\title{
Evaluation of the Controlled Release Ability of Theophylline from Xanthan/Chondroitin Sulfate Hydrogels
}

\author{
Ana-Maria Oprea ${ }^{1}$, Manuela-Tatiana Nistor ${ }^{1}$, Lenuta Profire ${ }^{3}$, Marcel Ionel Popa ${ }^{2}$, \\ Catalina Elena Lupusoru ${ }^{3}$, Cornelia Vasile ${ }^{1^{*}}$ \\ ${ }^{1}$ Department of Physical Chemistry of Polymers, Romanian Academy, "P. Poni” Institute of Macromolecular Chemistry, Iasi, Roma- \\ nia; ${ }^{2}$ Faculty of Chemical Engineering and Environmental Protection, "Gh. Asachi” Technical University, Iasi, Romania; ${ }^{3}$ Depart- \\ ment of Pharmacology and Algesiology, "Gr. T. Popa” Medicine and Pharmacy University, Iasi, Romania. \\ Email: *cvasile@icmpp.ro
}

Received January $14^{\text {th }}, 2013$; revised February $28^{\text {th }}, 2013$; accepted March $30^{\text {th }}, 2013$

Copyright (C) 2013 Ana-Maria Oprea et al. This is an open access article distributed under the Creative Commons Attribution License, which permits unrestricted use, distribution, and reproduction in any medium, provided the original work is properly cited.

\begin{abstract}
The xanthan/chondroitin sulfate (X/CS) hydrogels, obtained by a crosslinking technique, were evaluated in vitro and in vivo as matrices for theophylline release. The influence of $\mathrm{pH}$ of simulated physiological media on the $\mathrm{X} / \mathrm{CS}$ swelling behaviour at $37^{\circ} \mathrm{C}$ was investigated. The hydrogels theophylline loading degree was evaluated by near infrared chemical imaging (NIR-CI) technique and confirmed also by FT-IR spectroscopy; the drug loading was about $77.5 \%$ based on PLS-DA prediction (Partial least squares-Discriminate Analysis). The release profiles of theophylline from X/CS hydrogels in simulated gastric fluid (SGF) and simulated intestinal fluid (SIF) depend on CS content. The release mechanisms were controlled by the drug solubility and ionic properties of the polymers. In vivo theophylline delivery was done by oral administration. Pharmacokinetic analysis revealed sustained-release characteristics for 50/50 X/CS theophylline-loaded formulation compared with raw theophylline which was rapidly absorbed, distributed and eliminated. A good in vitro-in vivo correlation was found.
\end{abstract}

Keywords: Polysaccharide; Hydrogels; Theophylline; Swelling; In Vitro/In Vivo Release; Pharmacokinetics

\section{Introduction}

Sustained release dosage forms extend the duration time of drug therapy, reduce side-effects and increase safety and patient compliance by reducing the frequency of dosing. Multiple daily administration of an immediate release dosage form results in patient non-compliance. To control and modulate drug release properties of drugs, retardant polymers including hydrophilic polymers such as chitosan, chondroitin sulfate, xanthan and other polysaccharides have been utilized in dosage forms. The hydrophilic polymers control drug release from dosage forms by hydrogelation. The retardation mechanism is based on the intramolecular hydrogelation of a hydrophilic polymer during swelling and can be affected by the ionic strength of the dissolution medium. It is well known that the $\mathrm{pH}$ of the gastrointestinal tract (GI tract) varies from $\mathrm{pH} 1$ to 3 in the stomach and increases to approxi-

"Corresponding author. mately pH $7-8$ in the colon. Furthermore, the $\mathrm{pH}$ of the stomach can fluctuate with food intake, as well as with the age and health of the patient.

Due to the differences in ionic strength of gastric and intestinal fluids, as well as wide variations and fluctuations in its $\mathrm{pH}$ of the GI tract, the in vitro and in vivo data for sustained release dosage forms may not always correlate [1].

Xanthan gum $(\mathrm{X})$ is an extracellular complex polysaccharide secreted by the microorganism Xanthomonas campestris consisting of a cellulose backbone and trisaccharide side chains containing glucuronic acids that give this polymer a negative charge [2]. It is totally degraded in the colon by resident bacteria [3]. Xanthan gum was used for controlled release in combination with other polysaccharides as konjac glucomannan [4], ethylcellulose [5], guar gum [6], hydroxypropyl methylcellulose [7,8], chitosan and mesquite seed gum [9]. Xanthan gum, as a hydrophilic polymer, was added to the formu- 
lations to increase the drug release since ibuprofen release from the ibuprofen/ethylcellulose matrices $(60 / 40$, $\mathrm{w} / \mathrm{w}$ ) was too slow ( $20 \%$ in 24 hours). Changing the xanthan gum concentration as well as its particle size modified the in vitro drug release. Increasing xanthan gum concentrations yielded a faster drug release due to a higher liquid uptake, swelling and erosion rate [10].

Natural polysaccharides such as xanthan gum, xylan and guar gum are not digested in the human stomach or small intestine, but are degraded in the colon by resident bacteria [3] so that the drug remaining in matrix will be finally release by degradation/erosion of the matrix when drug delivery systems found suitable conditions. Because of the presence of the biodegradable enzymes only in the colon, the use of biodegradable polymers for colon specific drug delivery seems to be a more site specific approach as compared to other approaches. These types of matrices could shield the drug from the environments of stomach and small intestine and are able to deliver the drug to the colon. On reaching the colon, they undergo assimilation by microorganisms or degradation by enzyme or break down of the polymer backbone.

Drug release from the mini-matrices was mainly diffusion controlled, but swelling played an important role to obtain complete drug release. Drug release was influenced by the ionic strength of the medium as the conformation of xanthan gum molecules is determined by the salt concentration.

Chondroitin sulfate (CS) is one of the glycosaminoglycans which consists in repeating disaccharide units of Dglucuronic acid and $\mathrm{N}$-acetyl galatosamine sulfated at either 4- or 6-positions having a negative charge [11]. It is a biological polymer that is abundant in the extracellular matrix (ECM) of tissues throughout the body. CS can be used in various formulations, in combination with natural $[12,13]$ or synthetic polymers [14] as component of the drug release systems.

Theophylline, an alkaloid found in Camellia sinensis leaves is an effective drug used in the treatment of asthma and pulmonary disease and has been widely used as a model drug in various controlled release studies.

The aim of our study was to investigate the influence of the swelling/dissolution medium on the swelling behaviour of the xanthan/chondroitin sulfate hydrogels and the release mechanism of theophylline (THP) from these hydrogels. Correlation between in vitro and in vivo testing has been also followed.

\section{Materials and Methods}

\subsection{Materials}

Xanthan (X) with an average molecular weight of $2 \times 10^{6}$ $\mathrm{g} / \mathrm{mol}$ was purchased from Sigma-Aldrich, Germany.
Chondroitin sulfate (CS) was purchased from Roth, Germany. It was obtained from bovine tracheal cartilage. Epichlorohydrin used as crosslinking agent of analytical purity was purchased from Sigma-Aldrich and theophylline from Merck, Germany.

Preparation of xanthan/chondroitin sulfate hydrogels and their characterization were described in detail in a previous paper [15]. Briefly, the hydrogel samples were prepared in various weight mixing ratios ( $\mathrm{wt} \%)$ as: $90 / 10$, 80/20, 70/30, 60/40, 50/50 X/CS. Freshly distilled epichlorohydrin was added to the xanthan/chondroitin sulfate mixtures under vigorously continuous stirring. The samples gels-like were lied on glass plates and then the cross linking reaction was performed for 8 hours at $80^{\circ} \mathrm{C}$. Hydrogels were extensively washed with warm water to remove unreacted compounds especially epichlorohydrin. Obtained hydrogels were undergone to freeze drying for 10 hours by means of a LABCONCO FreeZone device.

\subsection{Investigation Methods for Hydrogels}

\subsubsection{Swelling Tests}

Swelling studies were carried out by direct immersion in simulated gastric fluid (SGF) $(\mathrm{pH} 2.2)$ and simulated intestinal fluid (SIF) (pH 7.4) at $37^{\circ} \mathrm{C}$ as in vivo experiments were done by oral administration. SGF was obtained by adding small $\mathrm{HCl}$ drops $(37 \mathrm{wt} \%$ ) in a certain quantity of water and SIF consisted in a PBS solution based on mixing $1.19 \mathrm{~g}$ of $\mathrm{KH}_{2} \mathrm{PO}_{4}$ and $4.3 \mathrm{~g} \mathrm{Na}_{2} \mathrm{HPO}_{4}$. The hydrogel samples were periodically removed from the solutions, gently wiped with a soft tissue to remove the excess surface solution, weighed and than carefully placed back into the vessel as quickly as possible. The reported weight of each sample was an average value of three measurements. The swelling degree (S) was calculated according to the equation:

$$
\mathrm{S}(\%)=\left(\mathrm{W}_{\mathrm{t}}-\mathrm{W}_{\mathrm{d}}\right) / \mathrm{W}_{\mathrm{d}} \times 100
$$

where $\mathrm{W}_{\mathrm{t}}$ is the weight of the swollen samples at time $\mathrm{t}$ and $\mathrm{W}_{\mathrm{d}}$ is the weight of the dry sample.

\subsubsection{FT-IR Spectroscopy}

The hydrogels loaded with theophylline were analyzed by FT-IR spectroscopy, using the $\mathrm{KBr}$ pellet technique. The spectra were scanned on a Bruker VERTEX 70 (USA) device, over the $4000-500 \mathrm{~cm}^{-1}$ range, at a resolution of $4 \mathrm{~cm}^{-1}$.

\subsubsection{Drug Loading}

The model drug used for in vitro and in vivo release measurements was theophylline. The loading was performed by a diffusion filling method which allows the partition of drug solution into the hydrogel network. Thus, $0.1 \mathrm{~g}$ 
$\mathrm{X} / \mathrm{CS}$ hydrogel in powder form was suspended in a $4 \%$ theophylline solution $(1: 1 \mathrm{v} / \mathrm{v}$, ethyl alcohol/water) under mild stirring and left to swell 24 hours, period corresponding with the time in which the compositions reached their equilibrium swelling degree, as determined from the swelling studies [16]. The loaded samples were then freeze-dried using a LABCONCO FreeZone device.

\subsubsection{Near Infrared Chemical Imaging Technique (NIR-CI Technique)}

NIR spectra of theophylline-loaded hydrogels were recorded on a SPECIM'S Ltd. SisuCHEMA controlled with Evince software package for processing the original image data. The system includes a Chemical Imaging Workstation for 1000 - $2500 \mathrm{~nm}$ NIR domains. The original image for each sample was taken with a NIR model spectral camera, respectively an imaging spectrograph type ImSpector N17E with 320 and 640 pixel spatial resolution at a rate of $60-350 \mathrm{~Hz}$.

\subsubsection{In Vitro Theophylline Release}

The in vitro release studies were performed both in SGF $(\mathrm{pH}$ 2.2) and SIF ( $\mathrm{pH}$ 7.4). During dissolution testing, the media was maintained at $37^{\circ} \mathrm{C} \pm 0.5^{\circ} \mathrm{C}$. Aliquots of the medium of $1 \mathrm{~mL}$ were withdrawn periodically at predetermined time intervals and analyzed using a HP 8450A UV-visible spectrophotometer at $\lambda=271 \mathrm{~nm}$, the wavelength characteristic to theophylline. In order to maintain the solution concentration the sample was carefully reintroduced in the circuit after analyzing. The concentrations of the drug were calculated based on calibration curves determined at the same wavelength.

A simple, semi-empirical equation using Korsmeyer and Peppas model was used to kinetically analyze the data regarding the drug release from studied matrices system which is applied at the initial stages (approximately $60 \%$ fractional release) [17]:

$$
\mathrm{M}_{\mathrm{t}} / \mathrm{M}_{\alpha}=\mathrm{k}_{\mathrm{r}} \mathrm{t}^{\mathrm{nr}}
$$

where $M_{t} / M_{\infty}$ represents the fraction of the released drug, $\mathrm{M}_{\mathrm{t}}$ and $\mathrm{M}_{\infty}$ are the cumulative amount of drug released at time $t$ and at infinite time (in this case maximum release amount in the experimental conditions used, at the plateau of the release curves), respectively, $\mathrm{k}_{\mathrm{r}}$ is a constant incorporating characteristics of the macromolecular matrix and of the drug: $n_{r}$ is the diffusion exponent, which suggests the release mechanism. In the equation above a value of $\mathrm{n}_{\mathrm{r}} \leq 0.5$ indicates a Fickian diffusion mechanism (sometimes tridimensional one) of the drug from matrix, while a value $0.5<\mathrm{n}_{\mathrm{r}}<1$ indicates anomalous or nonFickian behaviour. When $n_{r}=1$ a case II transport mechanism is involved while $n_{r}>1$ indicates a special case
II transport mechanism [18-20].

The corresponding drug-release profiles were represented through plots of the cumulative percentage of drug release versus time.

\subsubsection{In Vivo Theophylline Release}

Healthy Wistar rats weighing between 350 - 420 g purchased from Cantacuzino Institute, Bucharest, Romania were used for the study. The animals were maintained in identical laboratory conditions for one week before starting the experiments having access at food and water $a d$ libitum. In vivo tests were performed in accordance with the ethical rules stated in the paper "Ethical guidelines for investigations of experimental pain in conscious animals" [21].

The tested theophylline-loaded hydrogels have been orally delivered as suspensions in sodium carboxymethylcellulose of $0.5 \mathrm{wt} \%$. A dose of $15 \mathrm{mg}$ THP powder/ $\mathrm{kg}$ body weight was administrated for each rat. Blood samples were drawn out at established time intervals up to 72 hours. They were allowed to stand for 1 hour, centrifuged to separate the serum which was kept frozen $\left(-20^{\circ} \mathrm{C}\right)$ until further analysis. THP serum extraction was performed mainly as described in the literature [22] with a few minor changes. After thawing, $1 \mathrm{~mL}$ serum was mixed with $250 \mu \mathrm{l} 10 \mathrm{wt} \%\left(\mathrm{NH}_{4}\right)_{2} \mathrm{SO}_{4}$ solution and homogenized for 1 minute. THP was extracted for 15 minutes in $5 \mathrm{ml}$ 2-propanol:dichloromethane $(1: 9 \mathrm{v} / \mathrm{v}) \mathrm{mix}-$ ture. After 5 minutes centrifugation at $4000 \mathrm{rpm}$, the organic layer was drawn out and transferred into a glass tube and evaporated to dryness at $40^{\circ} \mathrm{C}$. The dry extract was dissolved in $1 \mathrm{~mL}$ acetonitrile $10 \mathrm{mM}$ aqueous sodium acetate $(7: 93 \mathrm{v} / \mathrm{v})$, passed through a $0.45 \mu \mathrm{m}$ syringe filter and used for further analysis. Plasma THP concentration was determined by high performance liquid chromatography (Shimadzu Model-CTO-20A HPLC system). The separation was performed on a $5 \mu \mathrm{m}$ ZORBAX SB-C18 column $(150 \times 4.6 \mathrm{~mm}$ i.d. $)$ under isocratic conditions with a mobile phase composed of acetonitrile $10 \mathrm{mM}$ aqueous sodium acetate $(7: 93 \mathrm{v} / \mathrm{v})$. Analyses were performed at room temperature under a flow rate of $1 \mathrm{~mL} / \mathrm{min}$ using an injection volume of $100 \mu \mathrm{l}$. THP was detected by UV detector at $270 \mathrm{~nm}$. THP concentration was determined with a calibration curve obtained with standard solutions of known THP concentrations in ethyl alcohol-water $(1: 1 \mathrm{v} / \mathrm{v})$ in the range of $1-20 \mu \mathrm{g} / \mathrm{mL}$, using HPLC software LC Solution Version 1.22 SP1 for integration and automatic determination of drug concentration in blood samples [16]. Each experiment was repeated four times and the mean value with standard deviation was reported. Statistical data analysis was performed using the Student $t$ test and ANOVA with $\mathrm{p}<$ 0.05 as the minimal level of significance. 


\section{Results and Discussion}

\subsection{Matrix Characterization}

\section{Swelling Studies in SGF and SIF}

The range of ionic strength $(\mu)$ in gastrointestinal (GI) fluid is about $0.010-0.166$ [23], but in $\mathrm{pH} 7.4$ may reach a value of $\mu 0.25$ [24]. The simulated medium used has a significant influence on $\mathrm{X} / \mathrm{CS}$ hydrogels swelling rate.

All X/CS hydrogels swelled, in SGF and SIF, very quickly during the first minutes (Figure 1); then the swelling rates of the $\mathrm{X} / \mathrm{CS}$ hydrogels became very slow, and finally reached the swelling equilibrium in about 100 minutes. These are typical biphasic swelling patterns, namely a rapid swelling followed by a slower sustained swelling (equilibrium swelling).

Time to reach the maximum swelling degree, in SGF, varies from 14 minutes in case of xanthan-based matrix with a degree of swelling about $727 \%$ to 6 minutes for composition 70/30 X/CS with a degree of swelling of $900 \%$, the process being much faster for the composition $50 / 50 \mathrm{X} / \mathrm{CS}$ with a maximum swelling degree of $1363 \%$ recorded in about 2 minutes (Table $\mathbf{1}$ ).

Equilibrium swelling degree values vary, also, with composition, the highest values being obtained for $60 / 40$

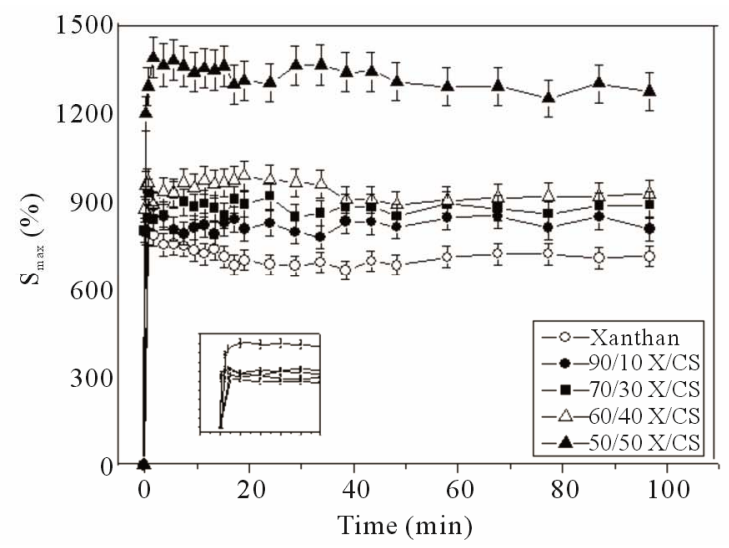

(a)
$\mathrm{X} / \mathrm{CS}$ and $50 / 50 \mathrm{X} / \mathrm{CS}$ matrices in both swelling media being of about $982 \%$ and $1381 \%$ in SGF and $1495 \%$ and $1642 \%$ in SIF, respectively.

Table 1 summarizes data on the swelling process characteristics in SGF and SIF of X/CS hydrogels with different mixing ratios at $37^{\circ} \mathrm{C}$.

For $\mathrm{X} / \mathrm{CS}$ hydrogels swelled in SIF the maximum swelling degree $\left(\mathrm{S}_{\max }\right)$ varies from $1067 \%$ for xanthanbased composition to $1426 \%$ and $1595 \%$ for $60 / 40 \mathrm{X} / \mathrm{CS}$ and $50 / 50 \mathrm{X} / \mathrm{CS}$, respectively and the values of time to reach maximum swelling degree $\left(\mathrm{t}_{\mathrm{max}, \mathrm{sw}}\right)$ decrease from 10 minutes for xanthan-based hydrogels to about $6 \mathrm{~min}$ utes for $70 / 30 \mathrm{X} / \mathrm{CS}, 60 / 40 \mathrm{X} / \mathrm{CS}$ and $50 / 50 \mathrm{X} / \mathrm{CS}$ compositions (Table 1).

Swelling rate $\left(\mathrm{k}_{\mathrm{sw}}\right)$ increases, in SGF, with $400 \% / \mathrm{min}$ ute for xanthan-containing and 90/10 X/CS compositions reaching a value of $1043 \%$ /minute for $50 / 50 \mathrm{X} / \mathrm{CS}$ matrix and in SIF is about $500 \%$ /minute for $90 / 10 \mathrm{X} / \mathrm{CS}, 80 / 20$ $\mathrm{X} / \mathrm{CS}$ and $70 / 30 \mathrm{X} / \mathrm{CS}$ reaching a value of $673 \%$ for $50 /$ $50 \mathrm{X} / \mathrm{CS}$ matrix (Table 1)

Half-swelling time $\left(\mathrm{t}_{1 / 2, \mathrm{sw}}\right)$ values obtained for $\mathrm{X} / \mathrm{CS}$ compositions vary in range between 41 - 10 seconds in SGF decreasing with increasing CS content in hydrogels compositions and in SIF is about 67 seconds for all matrices.

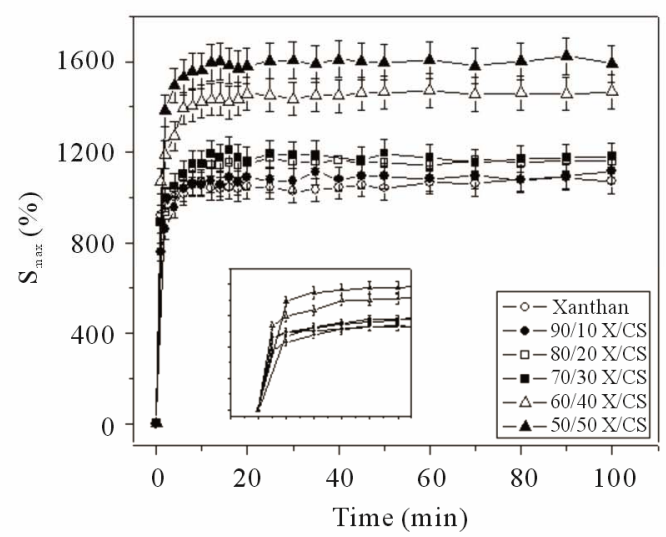

(b)

Figure 1. Swelling profiles of X/CS hydrogels with different compositions in medium of pH 2.2 (a) and pH 7.4 (b).

Table 1. The characteristics of the swelling process in SGF and SIF of $\mathrm{X} / \mathrm{CS}$ hydrogels of different composition at $37^{\circ} \mathrm{C}$.

\begin{tabular}{|c|c|c|c|c|c|c|c|c|}
\hline \multicolumn{5}{|c|}{ SGF } & \multicolumn{4}{|c|}{ SIF } \\
\hline $\mathrm{X} / \mathrm{CS}$ hydrogels $(\%)$ & $\mathrm{S}_{\max }(\%)$ & $\mathrm{T}_{1 / 2, \mathrm{sw}}(\mathrm{sec})$ & $\mathrm{t}_{\max , \mathrm{sw}}(\min )$ & $\mathrm{k}_{\mathrm{sw}}(\% / \mathrm{min})$ & $\mathrm{S}_{\max }(\%)$ & $\mathrm{T}_{1 / 2, \mathrm{sw}}(\mathrm{sec})$ & $\mathrm{t}_{\max , \mathrm{sw}}(\min )$ & $\mathrm{k}_{\mathrm{sw}}(\% / \mathrm{min})$ \\
\hline Xanthan & 727 & 41 & 14 & 453 & 1067 & 67 & 10 & 430 \\
\hline $90 / 10$ & 792 & 41 & 11.6 & 482 & 1119 & 67 & 7.9 & 494 \\
\hline $80 / 20$ & - & - & - & - & 1161 & 67 & 7.2 & 495 \\
\hline $70 / 30$ & 900 & 15 & 6 & 762 & 1181 & 67 & 6.4 & 499 \\
\hline $60 / 40$ & 944 & 15 & 3.8 & 800 & 1426 & 67 & 6.2 & 595 \\
\hline $50 / 50$ & 1363 & 10 & 1.8 & 1043 & 1595 & 67 & 5.9 & 673 \\
\hline
\end{tabular}


The swelling profiles of xanthan-containing hydrogels in SGF and SIF indicated that the swelling degree values in acidic medium are lower than in phosphate buffer solution. It is obvious that, being an acidic polymer with a $\mathrm{pK}_{\mathrm{a}}$ of 3.1, xanthan became less soluble at such a low $\mathrm{pH}$ value. From this result it can be predicted that swelling of this polysaccharide and of xanthan-containing hydrogels will be different in the stomach and intestine.

The X/CS hydrogels are superabsorbant and because the swelling process is very fast, the kinetic parameters such as $\mathrm{n}_{\mathrm{sw}}$ - exponent describing the mode of the penetrant transport mechanism and $\mathrm{k}_{\mathrm{sw}}$ - characteristic rate constant of the material describing the rate of swelling do not fit to any known transport mechanisms. The values of $\mathrm{n}_{\mathrm{sw}}$, obtained both in SGF and SIF, are between 0.06 and 0.14 which indicated that the transport mechanism was tridimensional Fickian diffusion [19], but very low values of $n$ are still controversed. If $n_{s w}$ is almost constant for all hydrogel compositions, $\mathrm{k}_{\mathrm{sw}}$ values increase with the amount of CS in hydrogel composition.

\subsection{Theophylline-Loaded Hydrogels Characterization}

\subsubsection{FT-IR Analysis}

FT-IR spectra of X/CS hydrogels loaded with theophylline are shown in Figure 2.

The band appearing at $3121 \mathrm{~cm}^{-1}$ is assigned to the aromatic $\mathrm{C}-\mathrm{H}$ stretching vibrations, whereas that appearing at $2922 \mathrm{~cm}^{-1}$ corresponding to aliphatic C-H stretching vibrations. The peaks appearing in region 2824 $2712 \mathrm{~cm}^{-1}$ are attributed to $\mathrm{N}=\mathrm{CH}$ bond.

The C-N stretching vibrations are seen at $1049 \mathrm{~cm}^{-1}$, while the one that appeared at $1243 \mathrm{~cm}^{-1}$ is assigned to aromatic $\mathrm{C}=\mathrm{O}$ stretching vibrations.

A slight shift of bands position from $1717 \mathrm{~cm}^{-1}$ to $1720 \mathrm{~cm}^{-1}$ and from $1667 \mathrm{~cm}^{-1}$ to $1670 \mathrm{~cm}^{-1}$ attributed to -CO-N(R)-CO- theophylline characteristic group con-

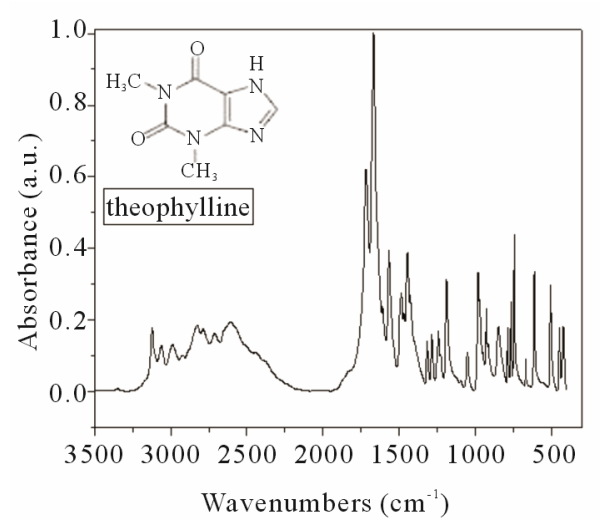

(a) firms presence of the theophylline in hydrogels and also the interactions between drug and hydrogels.

Some absorption bands could be influenced also by drug-hydrogels interactions such as slight shift of bands position to $1567 \mathrm{~cm}^{-1}$ and $1314 \mathrm{~cm}^{-1}$.

\subsubsection{NIR-CI Studies}

Theophylline distribution into X/CS hydrogels and its homogeneity were evaluated by the near infrared chemical imaging (NIR-CI) technique. The prediction of loading degree was evaluated based the new near infrared chemical imaging maps. The chemical imaging provides a simple method for evaluating the spatial drug distribution. The non-destructive character and minimal sample preparation required, show the feasibility of this technique which is used to investigate samples with pharmaceutical applications.

Both PLS-DA (Partial Least Squares-Discriminate Analysis) and PCA (Principal Component Analysis) models were used to determine the homogeneity and distribution of the two components, namely the drug and polymeric matrix. PLS-DA model based on multivariate inverse least squares discrimination method is used by Evince to classify the components. This could be achieved because of these two models based of mathematical processing. Assigning a colour for each principal component allows visual assessment of the degree of homogeneity of the components. As noted with a value between 0 and 1 of the same original component facilitated obtaining quantitative information. For the first component value 1 represents an available percentage of $100 \%$ and the value tends to zero when there is an unknown structure. The correct information can be extracted from the cube of information. The final images have for every pixel a complete spectrum that includes contributions from all the chemical components present in system.

Figure 3 corresponds to the PLS-DA model for 50/50

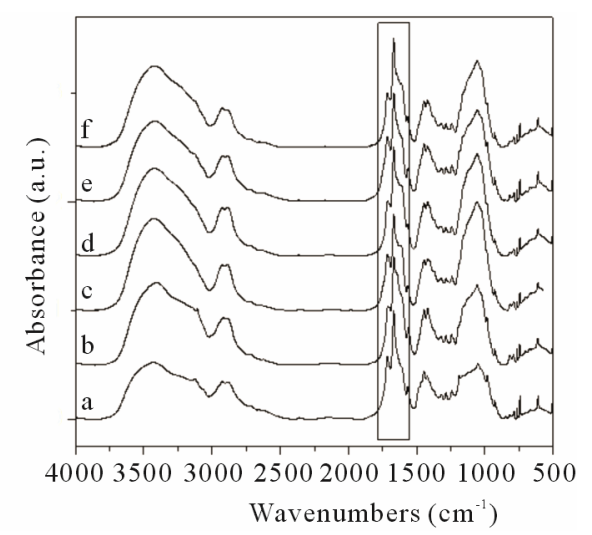

(b)

Figure 2. FT-IR spectra of theophylline (a) and X/CS hydrogels loaded with theophylline: (a) Xanthan-based hydrogel, (b) 90/10 X/CS, (c) 80/20 X/CS, (d) 70/30 X/CS, (e) 60/40 X/CS, (f) $50 / 50 \mathrm{X} / \mathrm{CS}$. 
$\mathrm{X} / \mathrm{CS}$ composition.

A uniform distribution was observed corresponding to a high homogeneity degree of the drug distribution in the $\mathrm{X} / \mathrm{CS}$ hydrogel matrix. Based on PLS-DA prediction, a theophylline loaded amount up to $77.5 \%$ into the $50 / 50$ $\mathrm{X} / \mathrm{CS}$ composition was found.

The obviously overlapping between the polymers and theophylline bands in near infrared region (Table 2) empower for the use a multivariate regression for the simultaneous determination of drug content in samples.

The NIR data of Table 2 confirm also FT-IR spectra results.

\subsubsection{Theophylline in Vitro Release Studies}

The release profiles of theophylline from X/CS hydrogels in SGF and SIF are shown in Figure 4.

In vitro release study of theophylline from $\mathrm{X} / \mathrm{CS}$ hy-

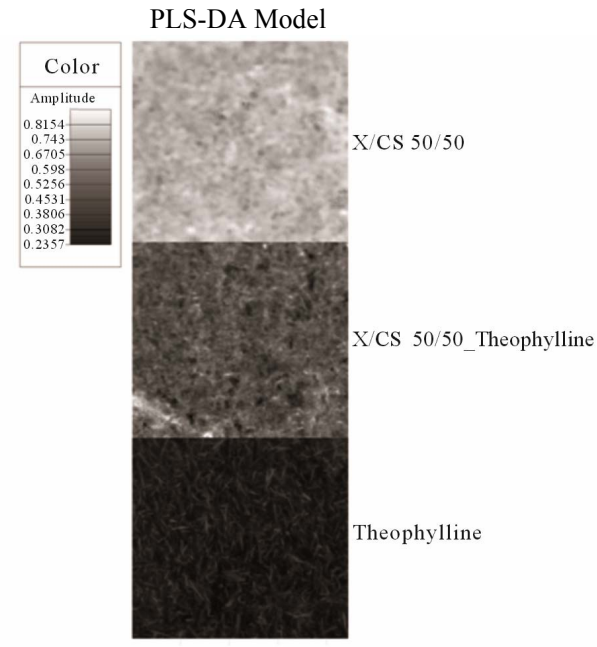

Figure 3. PLS-DA model for X/CS hydrogel.

Table 2. The wavelength corresponding to the functional groups on NIR spectra for 50/50 X/CS hydogel, theophylline and theophylline/50/50 X/CS system.

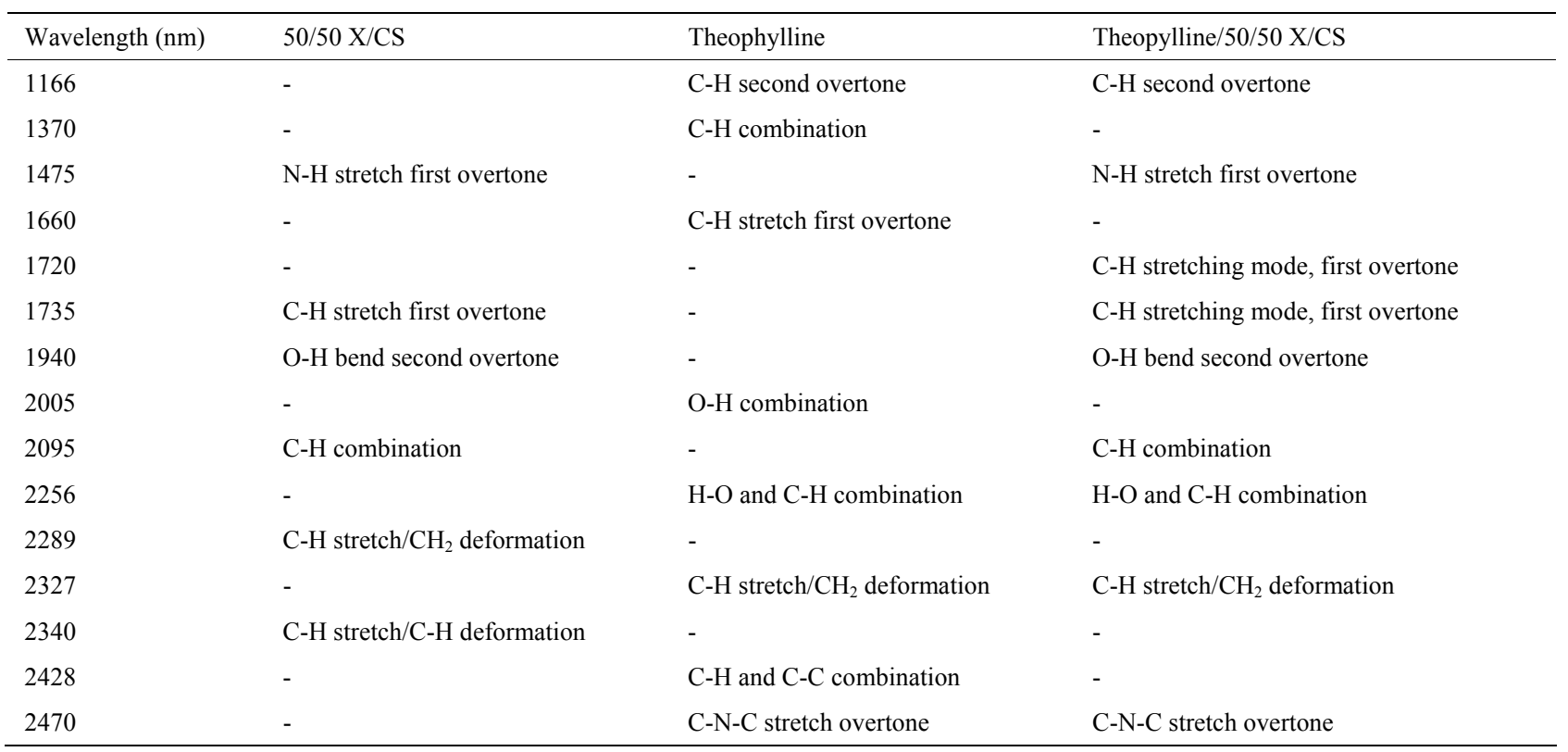

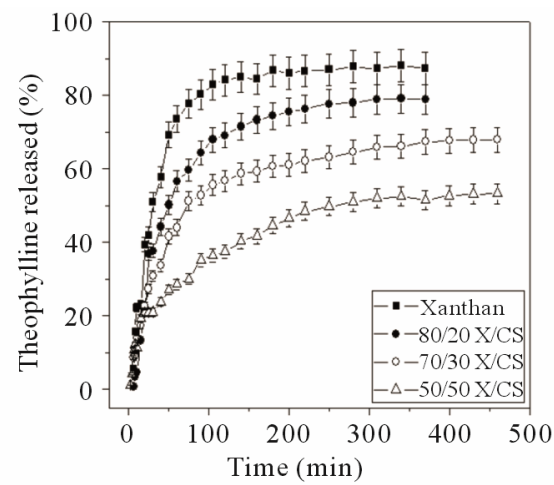

(a)

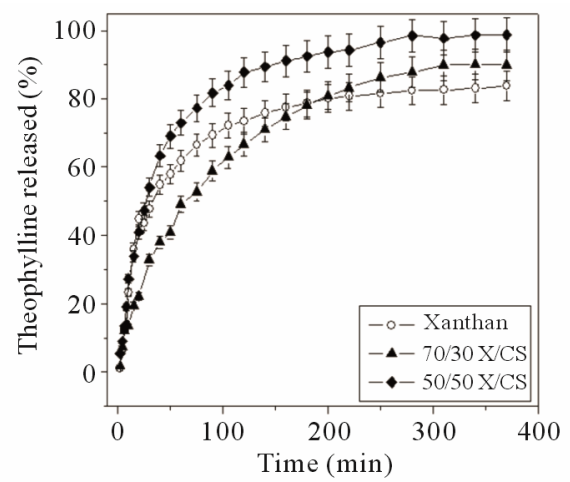

(b)

Figure 4. The release profiles of theophylline from X/CS hydrogels in SGF (a) and SIF (b) at $37^{\circ} \mathrm{C}$. 
drogels with different compositions was performed in SGF and SIF media at $37^{\circ} \mathrm{C}$.

In both cases, the release profiles of theophylline from $\mathrm{X} / \mathrm{CS}$ hydrogels depend on CS content.

In SGF, an increase of CS content leads to a decrease of theophylline percent released from $87 \%$ in case of xanthan-based hydrogel to $78 \%$ for $80 / 20 \mathrm{X} / \mathrm{CS}$ composition, $67 \%$ for $70 / 30 \mathrm{X} / \mathrm{CS}$ matrix reaching up to $53 \%$ in case of 50/50 X/CS hydrogel (Figure 4(a) and Table 3).

Half-release time $\left(t_{1 / 2}\right)$ and time to reach maximum percent released $\left(\mathrm{t}_{\max }\right)$ values from $50 / 50 \mathrm{X} / \mathrm{CS}$ composition are about two times higher than those obtained for xanthan-based matrix, namely theophylline half-release time values for xanthan-based composition is about 25 minutes and for $50 / 50 \mathrm{X} / \mathrm{CS}$ matrix is about 49 minutes. Time to reach maximum percent released $\left(\mathrm{Q}_{\max }\right)$ varies between 220 minutes for xanthan-based hydrogel to 400 minutes in case of 50/50 X/CS composition (Table 3).

It can be concluded that the release behaviour of theophylline in SGF from X/CS hydrogels depends on CS content so an increased percent of this component leads to a prolonged release process and small amount released. These results can be interpreted taking into consideration the dimensional and physical changes of the polymeric matrix during swelling according to the results obtained by other authors [25]. Substantial water uptake and gelling of xanthan gum appears to be responsible for sustained drug release [26].

The release studies performed in SIF showed that the maximum percentage cumulative release $\left(\mathrm{Q}_{\max }\right)$ is higher in case of 50/50 X/CS formulation than 70/30 X/CS and xanthan-based hydrogels (the trend is: $50 / 50 \mathrm{X} / \mathrm{CS}>$ 70/30 X/CS > xanthan-based hydrogel) (Table 3).

Half release time $\left(t_{1 / 2}\right)$ and time to reach maximum percent released $\left(t_{\max }\right)$ values decrease with increasing CS content in hydrogels compositions from 59 to 25.36 minutes and 340 to 280 minutes, respectively (Table 3).

The in vitro drug release experiments performed in SIF medium indicated the formation of a firmer gel structures and increased drug release rates in this medium.

\subsubsection{Kinetic Study of Theophylline Release}

The kinetic parameters for theophylline released in SGF and SIF from X/CS-based hydrogels are showed in Table 3 .

The release mechanism of theophylline in SGF and SIF is described by an anomalous transport for all tested formulations. In SGF the $n_{r}$ values decreased and in SIF increased with increasing CS content in hydrogel composition. The same behaviour was observed for the release rate constant values.

\subsubsection{In Vivo Release Profiles of Theophylline}

The raw theophylline and theophylline-loaded hydrogel have been orally delivered in rats as suspensions in sodium carboxymethyl cellulose of $0.5 \mathrm{wt} \%$. A dose of 15 mg THP powder/kg body weight was administrated for each rat. The chosen hydrogel composition for in vivo release study of theophylline was 50/50 X/CS which showed a sustained release during in vitro testing in SGF.

Figure 5 presents the pharmacokinetic release profiles of raw theophylline and theophylline-loaded in 50/50 X/ CS formulation.

The in vivo release profiles showed the sustained release of theophylline loaded in 50/50 X/CS formulation compared with raw theophylline which is removed from the body in the first 17 hours from administration. The theophylline released from 50/50 X/CS hydrogel showed a prolonged plasma concentration profile than raw theophylline, traces of active principles being recorded up to 20 hours after administration.

The evaluated pharmacokinetic parameters are given in Table 4.

The mean plasma level of raw theophylline was recorded at a $\mathrm{C}_{\max }$ value of $10.46 \mu \mathrm{g} / \mathrm{ml}$ at $\mathrm{t}_{\max }$ of 4 hours, while the $t_{1 / 2}$ is about 4.9 hours which indicated a fast absorption of pure theophylline. The theophylline serum concentration, released from 50/50 X/CS composition, reached a maximum value of $\mathrm{C}_{\max }$ of $16.33 \mu \mathrm{g} / \mathrm{ml}$ with a $t_{\max }$ of 4 hours and $\mathrm{t}_{1 / 2}$ of 8.5 hours, exhibiting a delayed absorption in blood (Table 4).

Serum theophylline concentration decreased with a re-

Table 3. The kinetic parameters and release characteristics of theophylline from X/CS hydrogels evaluated according to Korsmeyer-Peppas equation.

\begin{tabular}{|c|c|c|c|c|c|c|c|c|c|c|c|c|}
\hline \multirow{3}{*}{$\mathrm{X} / \mathrm{CS}$ hydrogels $(\%)$} & \multicolumn{6}{|c|}{ SGF } & \multicolumn{6}{|c|}{ SIF } \\
\hline & \multirow[b]{2}{*}{$t_{1 / 2}(\min )$} & \multirow[b]{2}{*}{$\mathrm{t}_{\max }(\min )$} & \multirow[b]{2}{*}{$\mathrm{Q}_{\max }(\%)$} & \multicolumn{3}{|c|}{ Korsmeyer-Peppas equation } & \multirow[b]{2}{*}{$\mathrm{T}_{1 / 2}(\min )$} & \multirow[b]{2}{*}{$\mathrm{t}_{\max }(\min )$} & \multirow[b]{2}{*}{$\mathrm{Q}_{\max }(\%)$} & \multicolumn{3}{|c|}{ Korsmeyer-Peppas equation } \\
\hline & & & & $\mathrm{n}_{\mathrm{r}}$ & $\mathrm{R}$ & $\mathrm{k}_{\mathrm{r}}\left(\min ^{-\mathrm{nr}}\right)$ & & & & $\mathrm{n}_{\mathrm{r}}$ & $\mathrm{R}$ & $\mathrm{k}_{\mathrm{r}} * 10^{3}\left(\min ^{-\mathrm{nr}}\right)$ \\
\hline Xanthan & 25 & 220 & 87 & 0.97 & 0.97 & 0.03 & 59 & 340 & 83 & 0.58 & 0.97 & 6.16 \\
\hline $80 / 20$ & 30 & 250 & 78 & 0.78 & 0.99 & 0.03 & - & - & - & - & - & - \\
\hline $70 / 30$ & 43 & 370 & 67 & 0.69 & 0.98 & 0.02 & 55 & 310 & 90 & 0.52 & 0.97 & 6.69 \\
\hline $50 / 50$ & 49 & 400 & 53 & 0.65 & 0.98 & 0.01 & 25.4 & 280 & 97 & 0.88 & 0.99 & 28.66 \\
\hline
\end{tabular}




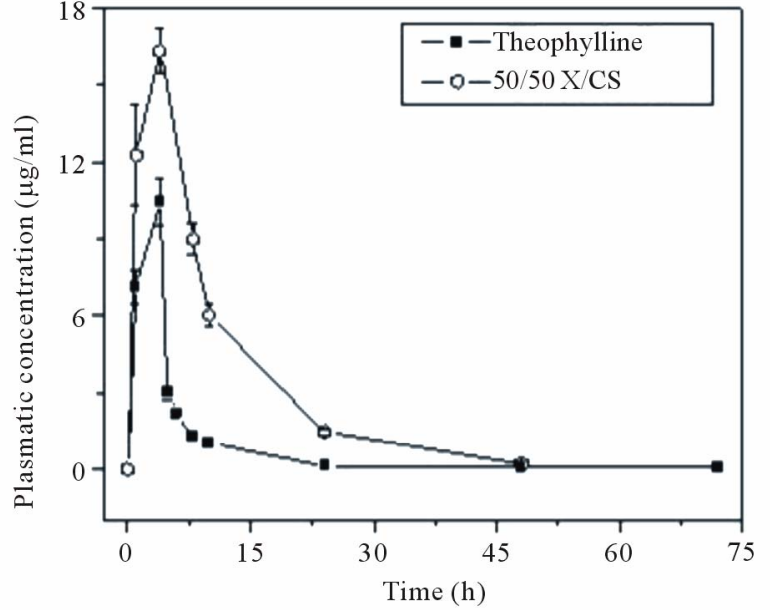

Figure 5. Plasma theophylline concentration versus time. Each point is presented as mean \pm S.D., $n=4$.

Table 4. Pharmacokinetic parameters obtained for raw theophylline and theophylline-loaded 50/50 X/CS hydrogel.

\begin{tabular}{lll}
\hline Parameters & $\begin{array}{l}\text { Raw } \\
\text { theophylline }\end{array}$ & $\begin{array}{l}\text { Theophylline-loaded } \\
50 / 50 \text { X/CS hydrogel }\end{array}$ \\
\hline $\mathrm{C}_{\max }(\mu \mathrm{g} / \mathrm{ml})$ & 10.46 & 16.33 \\
$\mathrm{t}_{\max }(\mathrm{h})$ & 4 & 4 \\
$\mathrm{t}_{1 / 2}(\mathrm{~h})$ & 4.9 & 8.5 \\
$\mathrm{AUC}_{0-72}(\mu \mathrm{g} \mathrm{h} / \mathrm{ml})$ & 58.11 & 187.77 \\
Relative bioavailability (\%) & - & 323 \\
\hline
\end{tabular}

latively low rate in 24 - 48 hours interval.

The value of area under the curve (AUC) or time dependence of plasma after administration of a single dose of drug was determined to be about $58.11 \mu \mathrm{g} \mathrm{h} / \mathrm{ml}$ for raw theophylline compared to $187.77 \mu \mathrm{g} \mathrm{h} / \mathrm{ml}$ for theophylline loaded in 50/50 X/CS formulation.

The relative bioavailability of $50 / 50 \mathrm{X} / \mathrm{CS}$ formulation loaded with theophylline compared to raw theophylline, both products containing the same dose of the same drug, is obtained by comparing their respective AUCs [27].

Relative bioavailability

$=\mathrm{AUC}_{\text {theophylline loaded }-50 / 50 \mathrm{X} / \mathrm{CS}} / \mathrm{AUC}_{\text {raw theophylline }}$

The significant differences between pharmacokinetic parameters may be due to the presence of chondroitin sulfate which increases the bioavailability about to $300 \%$ for the tested formulation and drug absorption through gastrointestinal mucosa without producing damage to the biological system, compared with raw theophylline.

The results obtained for in vivo therapeutic efficacy assessment of 50/50 X/CS hydrogel loaded with theophylline are correlated with those obtained for in vitro evaluation of the matrix in SGF. The sustained release ability of the theophylline, obtained both in vitro and in vivo, showed the qualities of this matrix; the presence of chondroitin sulfate in the composition causes the controlled and sustained behavior of release process.

\section{Conclusions}

The influence of the ionic strength on the theophyllineloaded release mechanism was evaluated in terms of pharmaceutical kinetics. Swelling of the xanthan/chondroitin sulfate matrix in acidic and buffer solutions showed that the ionic strength of the liquid has a strong effect on the sorptive properties of the matrix. From the release experiments, conducted at $37^{\circ} \mathrm{C}$, it was found that the drug delivery process was accomplished within the first 300 minute after immersion and theophylline was always released via a non-Fickian transport. The phenomenon can be described by a release by an equation with exponent $\left(\mathrm{n}_{\mathrm{r}}\right)$ varying from 0.65 to 0.97 depending on matrix composition. An increase of CS content leads to a decreased theophylline percent released in SGF in case of 50/50 $\mathrm{X} / \mathrm{CS}$ formulation and in SIF the theophylline releasing trend is: $50 / 50 \mathrm{X} / \mathrm{CS}>70 / 30 \mathrm{X} / \mathrm{CS}>$ xanthan-based hydrogel.

The results obtained in case of theophylline release studies performed in vitro, in SGF and in vivo are correlated, a sustained release ability of 50/50 X/CS matrix being revealed due to the presence of chondroitin sulfate in the composition.

\section{Acknowledgements}

The financial support from Romanian ANCS-UEFISCDI by PCCA Tip II BIONANOMED 164/2012 is gratefully acknowledged.

\section{REFERENCES}

[1] T. R. Reddy, D. Dhachinamoorthi and K. B. Chandrasekhara, "Independent Release Behavior of Glipizide Matrix Release Tablets Containing Chitosan and Xanthan Gum," International Journal of Pharmaceutical and Biomedical Research, Vol. 1, No. 2, 2010, pp. 64-70.

[2] J. V. Gruber, "Polysaccharide-Based Polymers in Cosmetics," In: E. D. Goddard and J. V. Gruber, Eds., Principles of Polymer Science and Technology in Cosmetics and Personal Care, Marcel Dekker, New York, 1999, pp. 339-403.

[3] A. A. Salyers, J. R. Vercellotti, S. E. West and T. D. Wilkins, "Fermentation of Mucin and Plant Polysaccharides by Strains of Bacteroides from the Human Colon," Applied and Environmental Microbiology, Vol. 33, No. 2, 1977, pp. 319-322.

[4] K. Wang, J. Fan, Y. Liu and Z. He, "Konjac Glucomannan and Xanthan Gum as Compression Coat for Colonic Drug Delivery: Experimental and Theoretical Evaluations," Frontiers of Chemical Engineering in China, Vol. 4, No. 1, 2010, pp. 102-108. 
doi:10.1007/s11705-009-0299-x

[5] T. Quinten, T. De Beer, F. O. Onofre, G. Mendez-Montealvo, Y. J. Wang, J. P. Remon and C. Vervaet, "Sustained-Release and Swelling Characteristics of Xanthan Gum/Ethylcellulose-Based Injection Moulded Matrix Tablets: In Vitro and in Vivo Evaluation," Journal of Pharmaceutical Sciences, Vol. 100, No. 7, 2011, pp. 28582870. doi:10.1002/jps. 22480

[6] J. Mor and A. Nanda, "Formulation and Evaluation of a Colon Targeted Drug Delivery System Using Ibuprofen as a Model Drug," The Pharmaceutical Research, Vol. 5, No. 1, 2011, pp. 176-183.

[7] W. Darunkaisorn and T. Phaechamud, "Sustained Release of Propranolol $\mathrm{HCl}$ Using Matrix Granule Comprising Wax and Polymers," Research Journal of Pharmaceutical, Biological and Chemical Sciences, Vol. 2, No. 1, 2011, pp. 158-166.

[8] M. M. Talukdar and R. Kinget, "Comparative Study on Xanthan Gum and Hydroxypropylmethyl Cellulose as Matrices for Controlled-Release Drug Delivery. II. Drug Diffusion in Hydrated Matrices," International Journal of Pharmaceutics, Vol. 151, No. 1, 1997, pp. 99-107. doi:10.1016/S0378-5173(97)04896-5

[9] C. César, S. Nogueira, L. M. Cabral, T. C. Dos Santos, A. Marucci and F. Alhaique, "Evaluation of New Polysaccharides Networks for Extended-Release Purposes: Mesquite Seed Gum (MSG), Xanthan Gum and Chitosan," Brazilian Journal of Pharmaceutical Sciences, Vol. 39, No. 3, 2003, pp. 273-288.

[10] E. Verhoeven, C. Vervaet and J. P. Remon, "Xanthan Gum to Tailor Drug Release of Sustained-Release Ethylcellulose Mini-Matrices Prepared via Hot-Melt Extrusion: In Vitro and in Vivo Evaluation," European Journal of Pharmaceutics and Biopharmaceutics, Vol. 63, No. 3, 2006, pp. 320-330. doi:10.1016/j.ejpb.2005.12.004

[11] C. T. Lee, P. H. Kung and Y. D. Lee, "Preparation of Poly(Vinyl Alcohol)-Chondroitin Sulphate Hydrogel as Matrices in Tissue Engineering," Carbohydrate Polymers, Vol. 61, 2005, pp. 348-354. doi:10.1016/j.carbpol.2005.06.018

[12] L. Huang, W. Sui, Y. Wang and Q. Jiao, "Preparation of Chitosan/Chondroitin Sulfate Complex Microcapsules and Application in Controlled Release of 5-Fluorouracil," Carbohydrate Polymers, Vol. 80, No. 1, 2010, pp. 168173. doi:10.1016/j.carbpol.2009.11.007

[13] K. Kofuji, T. Ito, Y. Murata and S. Kawashima, "Effect of Chondroitin Sulfate on the Biodegradation and Drug Release of Chitosan Gel Beads in Subcutaneous Air Pouches of Mice," Biological and Pharmaceutical Bulletin, Vol. 25, No. 2, 2002, pp. 268-271. doi: $10.1248 / \mathrm{bpb} .25 .268$

[14] E. C. Muniz, E. G. Crispim, A. R. Fajardo, J. F. Piai, E. R. F. Ramos, T. U. Nakamura, C. V. Nakamura and A. F. Rubira, "Hydrogels Based on Chemically Modified Poly (Vinyl Alcohol) (PVA-GMA) and PVA-GMA/Chondroitin Sulfate: Preparation and Characterization," Express Polymer Letters, Vol. 6, No. 5, 2012, pp. 383-395.

[15] A. M. Oprea, A. Neamtu and C. Vasile, "Xanthan/Chondroitin Sulfate Hydrogels as Carrier for Drug Delivery
Applications," Sectiunea Genetica si Biologie Moleculara, Vol. 12, No. 2, 2011, pp. 97-102.

[16] N. Popa, O. Novac, L. Profire, C. E. Lupusoru and M. I. Popa, "Hydrogels Based on Chitosan-Xanthan for Controlled Release of Theophylline," Journal of Materials Science: Materials in Medicine, Vol. 21, No. 4, 2010, pp. 1241-1248. doi:10.1007/s10856-009-3937-4

[17] R. W. Korsmeyer, S. R. Lusting and N. A. Peppas, "Solute and Penetrant Diffusion in Swellable Polymers. I. Mathematical Modeling," Journal of Polymer Science Part B: Polymer Physics, Vol. 24, No. 2, 1986, pp. 395408. doi:10.1002/polb.1986.090240214

[18] R. W. Korsmeyer and N. A. Peppas, "Solute and Penetrant Diffusion in Swellable Polymers. III. Drug Release from Glassy Poly (HEMA-co-NVP) Copolymers," Journal of Controlled Release, Vol. 1, No. 2, 1984, pp. 89-98. doi:10.1016/0168-3659(84)90001-4

[19] A. Kostić, B. Adnadjevic, A. Popovic and J. Jovanovic, "Comparison of the Swelling Kinetics of a Partially Neutralized Poly (Acrylic Acid) Hydrogel in Distilled Water and Physiological Solution," Journal of the Serbian Chemical Society, Vol. 72, No. 11, 2007, pp. 1139-1153. doi:10.2298/JSC0711139K

[20] L. Serra, J. Doménech and N. A. Peppas, "Drug Transport Mechanisms and Release Kinetics from Molecularly Designed Poly(Acrylic Acid-G-Ethylene Glycol) Hydrogels," Biomaterials, Vol. 27, No. 31, 2006, pp. 5440-5451. doi:10.1016/j.biomaterials.2006.06.011

[21] M. Zimmermann, "Ethical Guidelines for Investigations of Experimental Pain in Conscious Animals," Pain, Vol. 16, No. 2, 1983, pp. 109-110. doi:10.1016/0304-3959(83)90201-4

[22] N. Labačevski, D. Zendelovska, O. Sibinovska, S. Simeska, I. Kikerkov, P. Milosevski and S. Petrov, "Development and Validation of the HPLC Method for the Determination of Theophylline Serum Concentration: A Comparison with FPIA Method and Its Application for Bioequivalence Study," Bulletin of Chemists and Technologists of Macedonia, Vol. 22, No. 2, 2003, pp. 97-104.

[23] J. L. Johnson, J. Holinej and M. D. Williams, "Influence of Ionis Strength on Matrix Integrity and Drug Release from Hydroxypropyl Cellulose Compacts," International Journal of Pharmaceutics, Vol. 90, No. 2, 1993, pp. 151159. doi:10.1016/0378-5173(93)90151-5

[24] T. Solomon, "The Definition and Unit of Ionic Strength," Journal of Chemical Education, Vol. 78, No. 12, 2001, pp. 1691-1692. doi:10.1021/ed078p1691

[25] A. G. Andreopoulos and P. A. Tarantili, "Xanthan Gum as a Carrier for Controlled Release of Drugs," Journal of Biomaterials Applications, Vol. 16, No. 1, 2001, pp. 3446. doi:10.1106/XBFG-FYFX-9TW9-M83U

[26] M. C. Cohel and S. H. Bariya, "Fabrication of TripleLayer Matrix Tablets of Venlafaxine Hydrochloride Using Xanthan Gum," AAPS PharmSciTech, Vol. 10, No. 2, 2009, pp. 624-630. doi:10.1208/s12249-009-9244-Z

[27] R. Chereson, "Bioavailability, Bioeqiuvalence and Drug Selection," In: C. M. Makoid, P. J. Vuchetich and U. V. Banakar, Eds., Basic Pharmacokinetics, The Virtual University Press, 1996, pp. 1-111. 